\title{
A STUDY OF RETINAL NERVE FIBRE LAYER THICKNESS IN MYOPIA
}

\author{
Varsha Thomas ${ }^{1}$, Liby Joseph ${ }^{2}$
}

1Junior Resident, Department of Ophthalmology, Government Medical College, Kottayam, Kerala, India. ${ }^{2}$ Associate Professor, Department of Ophthalmology, Government Medical College, Kottayam, Kerala, India.

\section{ABSTRACT}

\section{BACKGROUND}

Myopia is a common refractive error of the eye and glaucoma is one of the potentially blinding ocular diseases associated with myopia. Assessment of the retinal nerve fiber laver (RNFL) thickness has been an important approach for detecting structural damage in patients with glaucoma. Although RNFL thinning is indicative of glaucomatous damage, it is uncertain whether RNFL thickness would vary with the refractive error of the eye. It is therefore important to investigate whether any correlation exists between RNFL measurements and the degree of myopia. We wanted to correlate peri papillary retinal nerve fibre layer (RNFL thickness with the degree of myopia.

\section{METHODS}

Visual acuity testing, automated refraction, retinoscopy were done in 75 consecutive patients diagnosed as myopia. SE was calculated and patients were arranged into 3 groups. Group 1 (low myopia), included all patients < 3 D, Group 2 included patients with -4 to -6 D and Group 3 were those with more than -6 D. OCT was done using TOPCON 3D OCT system for NFL thickness.

\section{RESULTS}

Among the 150 eyes analysed, significant RNFL thinning was noted in group 3 i.e., high myopes when compared to the other two groups and this difference was significant in the average RNFL thickness and also in all clock hour positions.

\section{CONCLUSIONS}

This study establishes that high myopes have a significantly different RNFL thickness profile. It has to be taken into consideration while diagnosing glaucoma in patients with myopia.

\section{KEY WORDS}

RNFL Thickness, OCT (Optical Coherence Tomography), Myopia

HOW TO CITE THIS ARTICLE: Thomas V, Joseph L. A study of retinal nerve fibre layer thickness in myopia. J. Evolution Med. Dent. Sci. 2019;8(28):2264-2269, DOI: 10.14260/jemds/2019/496

\section{BACKGROUND}

Myopia is a common refractive error of the eye. Its prevalence has increased markedly in recent years especially in Asian countries. Glaucoma is one of the potentially blinding ocular diseases associated with myopia.

Assessment of the Retinal Nerve Fibre Layer (RNFL) thickness has been an important approach for detecting structural damage in patients with glaucoma

The optical coherence tomography (OCT) is a noncontact and non-invasive modern imaging device designed to measure the RNFL thickness. OCT can directly measure and quantify RNFL thickness by calculating the area between the internal limiting membrane (ILM) and RNFL border. Although RNFL thinning is indicative of glaucomatous damage, ${ }^{1}$ it is uncertain whether RNFL thickness would vary with the refractive error of the eye in different clock hours of the optic disc. It is therefore important to investigate whether any correlation exists between RNFL measurements in different clock hours and degree of refractive error in myopia.

'Financial or Other Competing Interest': None.

Submission 03-04-2019, Peer Review 27-06-2019,

Acceptance 05-07-2019, Published 15-07-2019.

Corresponding Author:

Dr. Liby Joseph,

Associate Professor,

Department of Ophthalmology,

Government Medical College, Kottayam, Kerala, India.

E-mail: libydoctor@gmail.com

DOI: $10.14260 / j e m d s / 2019 / 496$

\begin{abstract}
Aim of the Study
To co-relate the average and clock wise peri papillary retinal nerve fibre layer (RNFL) thickness using Spectral Domain OCT in patients with varying degree of myopia.
\end{abstract}

\section{METHODS}

Study Design

Descriptive Study.

\section{Sample Size}

Was calculated on the basis of a study conducted by Kamath AR, Dudeja L and using the formula:

Sample size $=\left\{(\mathrm{z}\right.$ alpha $+\mathrm{z}$ beta $\left.) 2^{*} \mathrm{SD} 2 * 2\right\} / \mathrm{d} 2$. Applying the formula, the sample size reached was at least 39 patients in each group. So 50 eyes were included in this study in each group.

\section{Sampling Technique}

After obtaining the IEC/IRB clearance (74/2016), the study was commenced by including 150 eyes of first 75 OPD patients diagnosed with myopia.

\section{Inclusion Criteria}

1. Diagnosed cases of myopia attending the Ophthalmology.

2. OPD during the study period.

\section{Exclusion Criteria}

1. Patients diagnosed with glaucoma or with ocular hypertension.

2. Diagnosed cases of other optic neuropathies. 
3. Intra Ocular Pressure $>21 \mathrm{~mm} \mathrm{Hg}$.

4. Patients with history of previous trauma or ocular surgery.

5. Patient's with Neurodegenerative diseases ${ }^{4}$.

6. Any patients with significant media haziness precluding OCT scan.

7. Any retinal diseases other than myopia.

\section{Study Tools}

1. TOPCON 3 D OCT 1 (Spectral Domain OCT).

2. Slit lamp.

3. $90 \mathrm{D}$ lens.

4. Direct ophthalmoscope.

5. Ultrasound A SCAN (For axial length).

6. Retinoscope.

7. Goldman Applanation Tonometry.

8. TOPCON Auto Kerato Refractometer.

After taking proper informed consent, relevant history and clinical examination, all the exclusion criteria were checked for and any such cases were excluded. Using Snellen's chart visual acuity was assessed. Automated refraction and retinoscopy were done, and the refractive error noted and adjusted to spherical equivalent. Patients whose refraction measured up to -3 Dioptres were classified as Group 1, those between -3 to -6 D were classified as Group 2, and those with more than -6 D were classified as Group 3. The thickness of the peri-papillary NFL was measured using Spectral Domain Optical Coherence Tomography (TOPCON 3D OCT machine system. All scans were done by the same operator. The RNFL thickness in all clock hour positions, the average RNFL thickness and the average thickness in the four sectors: Superior, Inferior, Nasal and Temporal quadrants were noted.

\section{Degree of Refractive Error}

The mean spherical equivalent (S E) in group 1 was found to be -1.37 while that of group 2 was -4.5391 and that of group 3 was -8.3894 respectively with the mean of the whole group being -4.76 .

\section{Superior Quadrant RNFL Thickness}

The mean RNFL thickness of the superior quadrant in groups 1, 2 and 3 were 136,127.68, 109.18 microns respectively with the thickness decreasing as the myopia increases. Group 3 had the thinnest RNFL with the difference in thickness being significant with a p value of 0.00 on ANOVA and on comparison between groups the difference was significant between groups 1 and 3 ( $p$ value 0.00 ) and groups 2 and 3 ( $p$ value 0.00 )

\section{Inferior RNFL Thickness}

The average RNFL thickness in groups 1, 2 and 3 were 138.66, $129.68,107.14$ microns respectively with least thickness for group 3 and the decrease in RNFL thickness was significant with a $p$ value of 0.00 and on comparing $s$ a significant difference in RNFL thickness was found between group 1 and group 3 ( $p$ value 0.00 ) and group 2 and group 3 ( $p$ value 0.00 ).

\section{RESULTS}

Gender Distribution

Among the total 150 myopic patients studied 53 were males and 97 were females. Group 1 had 10 males and 40 females, group 2 had 22 males and 28 females while group 3 had 21 males and 29 females.

\begin{tabular}{|c|c|c|c|}
\hline & Male & Female & Total \\
\hline Group 1 & 10 & 40 & 50 \\
\hline Group 2 & 22 & 28 & 50 \\
\hline Group 3 & 21 & 29 & 50 \\
\hline Total & $\mathbf{5 3}$ & $\mathbf{9 7}$ & $\mathbf{1 5 0}$ \\
\hline \multicolumn{3}{|c|}{ Table 1. Gender Distribution of Myopes } \\
\hline
\end{tabular}

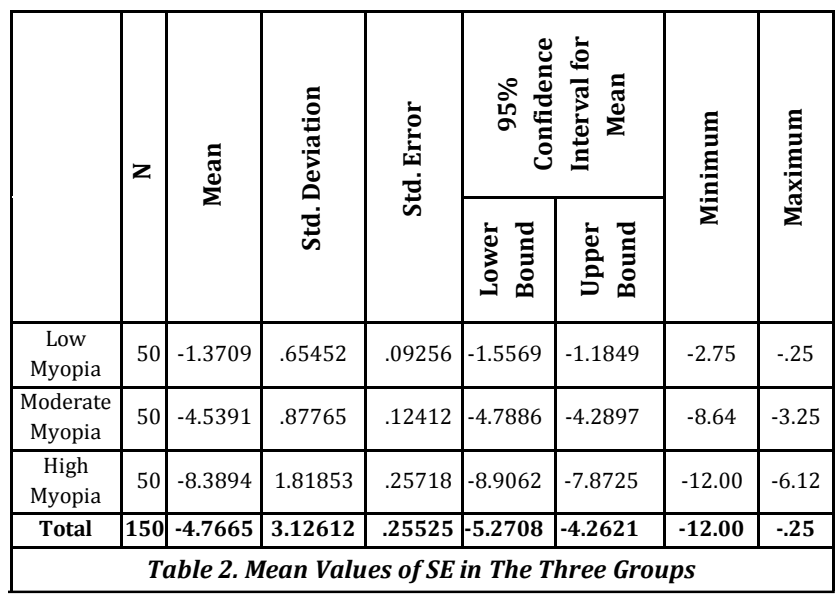
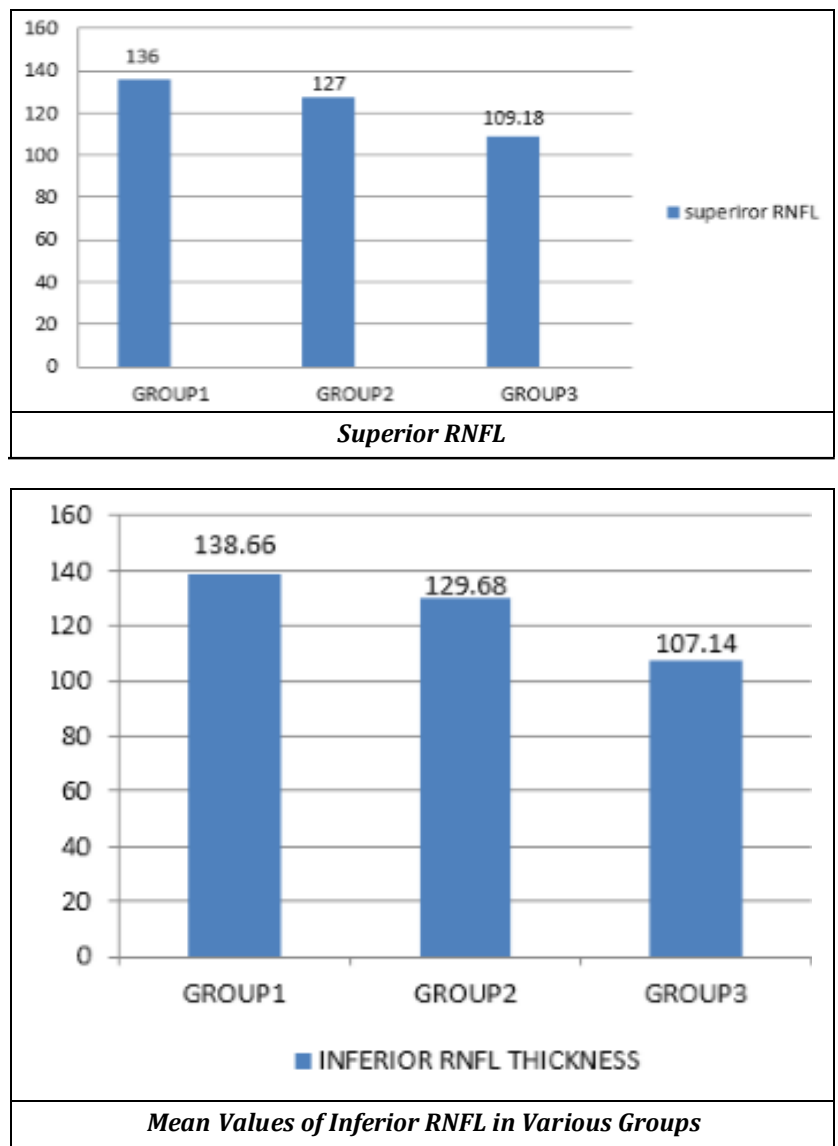

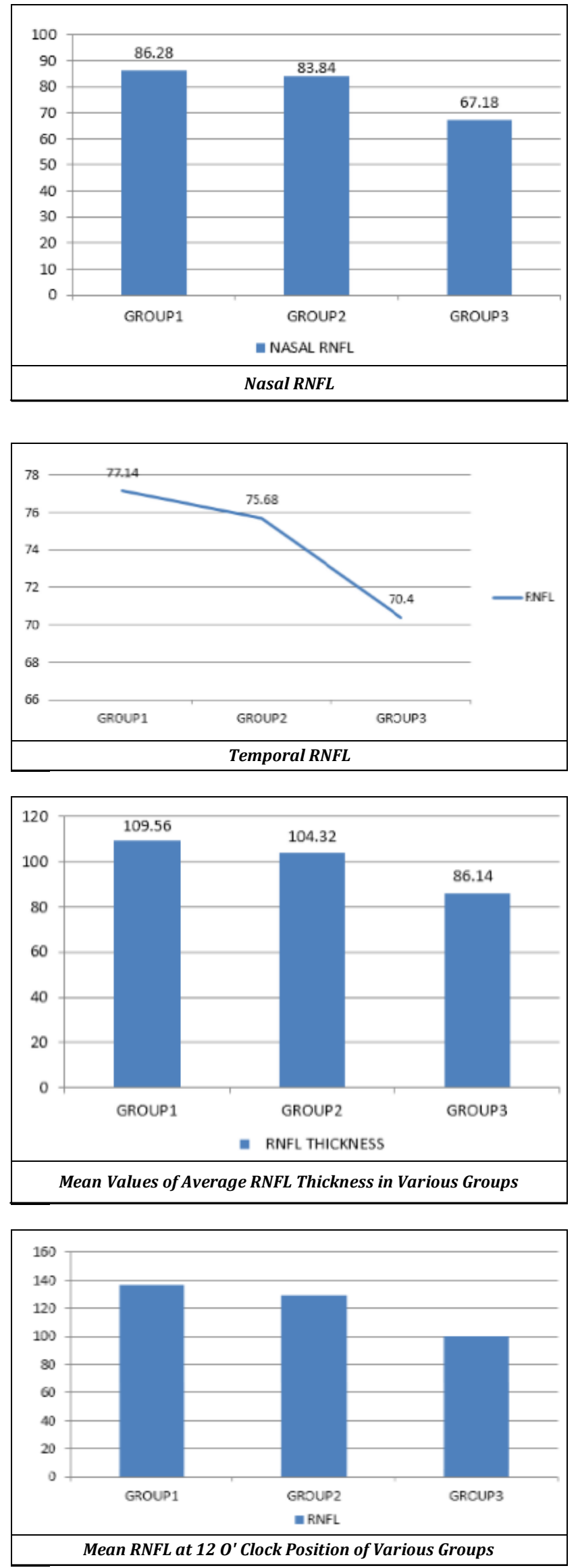
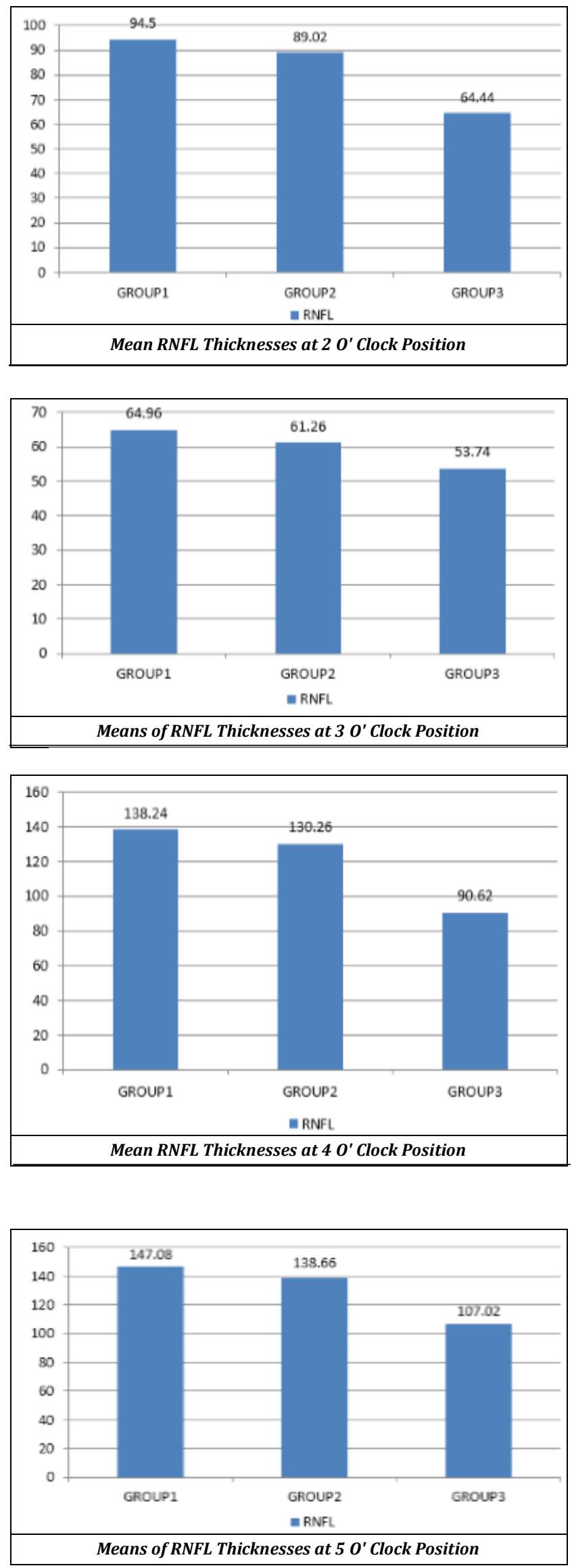

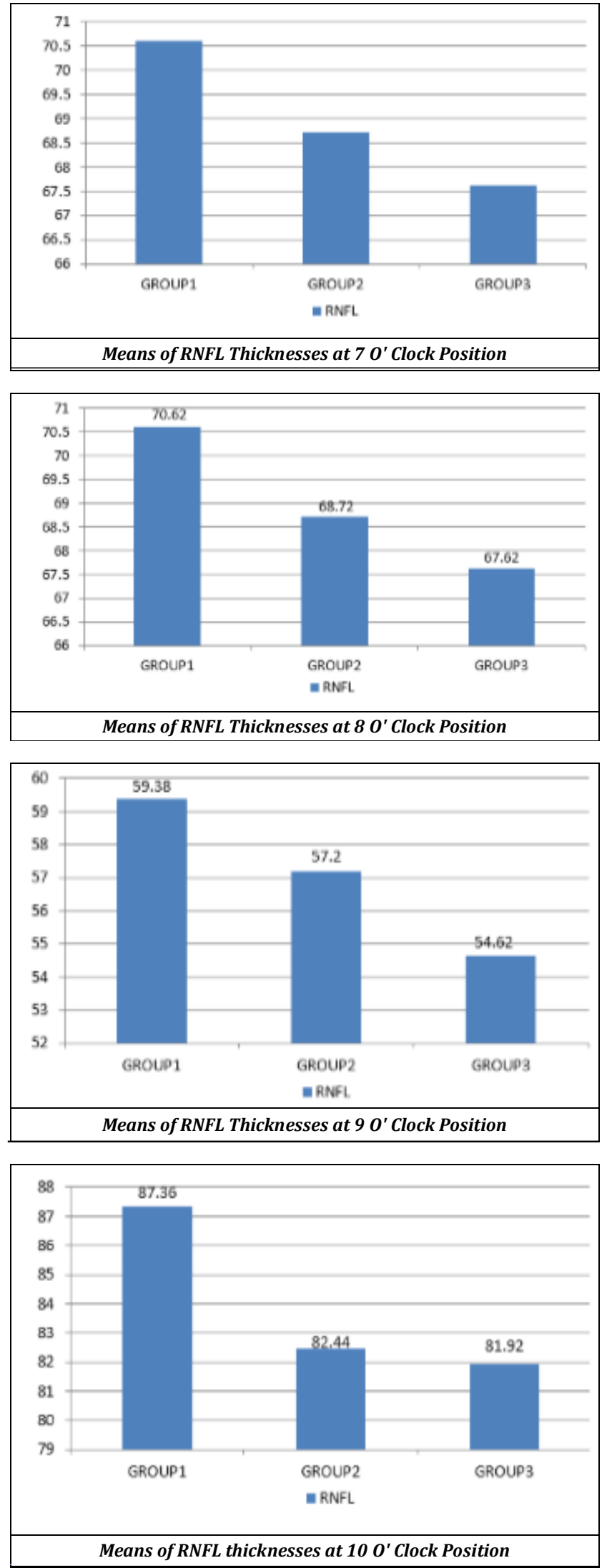

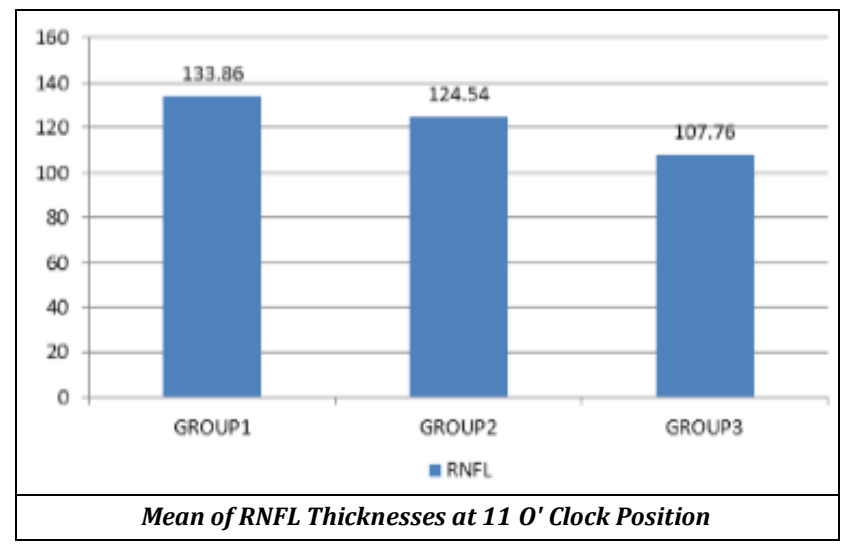

\section{Nasal RNFL Thickness}

The average RNFL thickness in the nasal quadrant of groups 1 , 2 and 3 were $86.28,83.84$ and 73.14 microns respectively with group 3 showing significant RNFL thinning when compared to the other 2 groups ( $p$ value 0.00 on comparing group 1 and group 3 and group 2 and group 3 ) and the difference between the three groups being significant with a $p$ value of 0.001 on ANOVA.

\section{Temporal RNFL Thickness}

The average RNFL thickness in the temporal quadrant in groups 1, 2 and 3 were 77.14, 75.68 and 70.4 microns respectively without any significant RNFL thinning with a $p$ value of 0.226 . On comparison between groups, there was no significant difference between groups 1 and 2(p value 0.961 ) or groups 2 and 3 ( $p$ value 0.371 ) or groups 2 and 3 ( $p$ value 0.242 )

\section{Average RNFL Thickness}

The average RNFL thickness was found to decrease with increase in the degree of myopia with group three having significant thinning of RNFL when compared to the other 2 groups. On testing the variables with ANOVA, a significant difference with a $p$ value of 0.00 was found out. On comparison between groups using the Tukeys test significant difference was noted between groups 1 and 3 ( $p$ value 0.00 ) and groups 2 and 3 , (p value 0.00 ) with no significant difference between groups 1 and 2 (p value 0.115 )

\section{Clockwise RNFL Thickness}

On studying the clockwise RNFL thickness, significant thinning was noted in group 3 as compared to the other 2 groups in all quadrants except 4,8,9 0' clock positions and the difference between the groups were significant in all positions except 4,8,9 0'clock positions with a p value < 0.05 in those cases. 


\section{a. 12 0' Clock}

The mean RNFL in groups 1,2 and 3 are 136.64,129.50,100.04 microns respectively with group 3 showing significant thinning when compared to groups 1 and 2 ( $p$ value 0.00 for each comparison by Tukey's test) and the difference between the three groups are significant on ANOVA with a p value of 0.00 .

\section{b. 1 0' Clock position}

The average thickness of RNFL in groups 1,2 and 3 are 136.6,132.4,93.6 respectively with group 3 showing significant thinning when compared to groups 1 ( $p$ value 0.00 ) and $2(p$ value 0.00$)$ and the difference between the three groups are significant on ANOVA with a $p$ value of 0.00

\section{c. 2 0' Clock position}

The mean RNFL thickness of groups 1,2 and 3 are 94.5,89.02 and 64.44 microns respectively with significant thinning noted in group 3 when compared to groups $l(p$ value 0.00$)$ and 2 ( $p$ value 0.000 ) and there was significant difference in RNFL thickness between the three groups on ANOVA with $p$ value 0.00

\section{d. 3 0' Clock Position}

The mean RNFL thickness of groups 1,2 and 3 are 64.96, 61.26 and 53.74 microns respectively with group 3 showing significant difference in RNFL thickness when compared to groups 1 ( $p$ value 0.000 and 2 ( $p$ value 0.000 ) and there is significant difference in RNFL thickness between these groups on ANOVA ( $p$ value 0.00 ).

\section{e) 4 0' Clock Position}

The mean RNFL thickness of groups 1, 2 and 3 are 79.48, 75 . 16 and 71.78 microns respectively with no significant difference in RNFL thickness between these groups on ANOVA ( $p$ value 0.085). On comparison between groups on Tukey's test also, no significant difference was found out between groups 1 and 2 ( $\mathrm{p}$ value 0.424 ) or groups 1 and 3 ( $\mathrm{p}$ value 0.069 ) or groups 2 and 3 (p value 0.590 )

\section{f. 5 O' Clock Position}

The mean RNFL thickness of groups 1,2 and 3 are 138.24,130.26 and 90.62 microns respectively with group 3 showing significant difference in RNFL thickness when compared to groups 1 ( $p$ value 0.000 and 2 ( $p$ value 0.000 ) and there is significant difference in RNFL thickness between the three groups on ANOVA (p value 0.000)

\section{g) 7 0' Clock Position}

The mean RNFL thickness of groups 1,2 and 3 are 133.44,120.24 and 105.08 microns respectively with Group 3 showing significant difference in RNFL thickness when compared to groups 1 ( $p$ value 0.000 ) and 2 ( $p$ value 0.000 ) and there is significant difference in RNFL thickness between the three groups with a $\mathrm{p}$ value of 0.00

\section{h) 8 0' Clock Position}

The mean RNFL thickness of groups 1, 2 and 3 are 70.62, 68 . 72 and 67.62 microns respectively with no significant difference in RNFL thickness between these groups ( $p$ value 0.597 on ANOVA).On Tukeys test no significant difference between these groups on comparing them with each other $(\mathrm{p}$ value on 0.8 on comparison between groups 1 and 2,p value 0.575 between 1 and 3 and a p value of 0.928 between 2 and 3 respectively).

\section{i) 9 0' Clock Position}

The mean RNFL thickness of groups 1, 2 and 3 are 59.38,57.12 and 54.62 microns respectively with no significant difference in RNFL thickness between these groups ( $p$ value 0.079 on ANOVA). On Tukey's test no significant difference between these groups on comparing them with each other ( $\mathrm{p}$ value 0.559 ) on comparison between groups 1 and 2,p value 0.063 between 1 and 3 and a p value of 0.432 between 2 and 3 respectively.

\section{j) 10 0' Clock Position}

The mean RNFL thickness of groups 1,2 and 3 are 87.36, 82.44 and 81.92 microns respectively with no significant difference in RNFL thickness between these groups ( $p$ value 0.129 on ANOVA). On Tukey's test no significant difference between these groups on comparing them with each other ( $p$ value 0.220 on comparison between groups 1 and $2, p$ value 0.158 between 1 and 3 and a p value of 0.983 between 2 and 3 respectively)

\section{k) 11 0' Clock Position}

The mean RNFL thickness of groups 1,2 and 3 are 133.86,124.54 and 107.76 microns respectively with group 3 showing significant difference in RNFL thickness when compared to groups 1 ( $p$ value 0.000 ) and 2 ( $p$ value 0.001 ) and there is significant difference in RNFL thickness between the three groups with a $p$ value of 0.00 on ANOVA.

\section{DISCUSSION}

The average peripapillary retinal nerve fibre layer thickness decreased as the refractive error increased and this was more significant in the high myopia group than in low and moderate myopia groups.

The retinal nerve fibre layer thickness significantly decreased in myopia $>6$ D. Significant decrease in peripapillary retinal nerve fire layer thickness occurred at 12, 1, 2, 3, 5, 6, 7 and 110 'clock positions with an insignificant change at the 4 , 8, 9 and 100 ' clock positions.

A study by Kamath AR, Dudeja $\mathrm{L}^{1}$ showed similar results in which among the total 118 myopic eyes studied, the eyes in Group 3 show significant thinning in the average RNFL thickness and in all quadrants except temporal as compared to Group 1 and 2. Correspondingly, in eyes with myopia > 6 D (as compared to Group 1 and 2), decrease in RNFL thickness is seen in all clock hour positions except 4, 8, 9, and 10 .

In another study by V. Sowmya, V. R. Venkataramanan, and Vishnu Prasad ${ }^{2}$ on effect of Refractive Status and Axial Length on Peripapillary Retinal Nerve Fibre Layer Thickness which included 300 eyes of 150 patients who underwent RNFL analysis using TOPCON 3D OCT 2000 showed that RNFL thickness average shows increasing thickness with increase in hypermetropia and decreasing thickness with increase in myopia. The change was found to be statistically significant when hypermetropia or myopia was $>3.0 \mathrm{D}$ when compared with normal group and the change in myopia was more significant $(p=0.001)$ than hypermetropia $(p=0.031)$. 
In another study by Mousumi Malakar, Syed Nasir Askari and Adil Asghar. ${ }^{3}$ on Optical Coherence Tomography Assisted Retinal Nerve Fibre Layer Thickness Profile in High Myopia, fifty myopic eyes (25 patients) and forty emmetropic eyes, were evaluated by Fourier domain optical coherence tomography (FD OCT). The overall mean RNFL thickness in the myopic groups and controls were 87.89 microns and 111.64 |microns respectively. The mean retinal nerve fibre thickness was significantly less in myopic eyes as compared to control group ( $p=0.0001)$. In all the quadrants the retinal nerve fibre thickness was significantly decreased in myopic eyes as compared to emmetropic eyes.

In a study by Taruna Chowdhary, Dr. D. S. Chowdhary, Anju Choudhary ${ }^{4}$ on Study of Retinal Nerve Fiber Layer Thickness in Myopic Eye in Rajasthani Population, the subjects were divided into two diagnostic groups, according to refractive error; Group A low myopia (Spherical equivalent between $0.50 \mathrm{D}$ to $-6.00 \mathrm{D}$ ) and Group B high myopia (spherical equivalent $-6.00 \mathrm{D}$ and more) and the results showed that average RNFL thickness decreased with increase in degree of myopia. Linear regression analysis shows positive correlation between RNFL thickness and degree of myopia $(\mathrm{r}$ value $=$ $0.5286, p=0.0001$ ). In contrast, Hoh et al ${ }^{5}$ suggested that the peri-papillary RNFL thickness does not vary with degree of myopia or axial length. Among studies based on stratus OCT, a study in 48 Korean subjects by Kim MJ et al ${ }^{6}$ found average RNFL to be significantly lower in high myopia than in low myopia group. In quadrant-wise measurements, RNFL thickness was thicker in low myopia group than in moderate or high myopia group for superior, inferior, and nasal quadrants (all $P$ values $<0.020$ ). However, temporal quadrant showed thinning in low myopia group than in moderate or high myopia groups. $(\mathrm{P}=0.001)$. The study supports the fact that the topographical profile of RNFL thickness in myopes especially high myopes is significantly different and shows significant thinning. So this should be considered before labelling a high myope as a glaucoma suspect based on OCT findings alone.

\section{CONCLUSIONS}

This study demonstrated that subjects with highly myopic eyes (myopia $>6 \mathrm{D}$ ) had thinner RNFL than subjects with low or moderate myopia. No significant difference was noted in RNFL thickness profile between low (Myopia < 3D) and moderate myopia groups (myopia 3-6D). This should be taken into consideration while analysing RNFL thickness for the diagnosis of glaucoma in subjects with highly myopic eyes.

\section{REFERENCES}

[1] Kamath AR, Dudeja L. Peri-papillary retinal nerve fiber layer thickness profile in subjects with myopia measured using optical coherence tomography. J Clin Ophthalmol \& Res 2014;2(3):131-6. (Cited 2016 Nov 22).

[2] Sowmya V, Venkatramanan VR, Prasad V. Effect of refractive status and axial length on peripapillary retinal nerve fibre layer thickness: an analysis using 3D OCT. J Clin Diagn Res 2015;9:NC01-4.

[3] Malakar M, Askari S, Asghar A. Optical coherence tomography assisted retinal nerve fibre layer thickness profile in high myopia. J Clin Diagn Res 2015;9(2):NC01-NC03.

[4] Choudhary T, Choudhary DS, Choudhary A. Study of RNFL thickness in myopic eyes in Rajasthani population. IJSR 2015;4(2):880-1.

[5] Hoh ST, Lim MC, Seah SK, et al. Peripapillary retinal nerve fiber layer thickness variations with myopia. Ophthalmology 2006;113(5):773-7.

[6] Kim MJ, Lee EJ, Kin TW. Peripapillary retinal nerve fibre layer thickness profile in subjects with myopia measured using the Stratus optical coherence tomography. Br J Ophthalmol 2010;94(1):115-20. 